\title{
PREHOSPITAL MEDICINE
}

\section{Outcome of diabetic patients treated in the prehospital arena after a hypoglycaemic episode, and an exploration of treat and release protocols: a review of the literature}

\section{K Roberts, A Smith}

See end of article for

authors' affiliations

Emerg Med J 2003;20:274-276

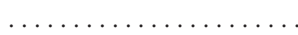

Correspondence to: Miss K Roberts, Pre-hospital Emergency Research Unit Lansdowne Hospital, Cardiff CF 11 8UL, UK;

ks.roberts@

emergency-research.co.uk

Accepted for publication 27 May 2002

Objectives: This review examines current treat and release protocols adopted by the ambulance service, and factors that may predispose patients to hypoglycaemia.

Methods: Online database searches and hand searches of journals led to 241 articles being found, of which eight were used for this article.

Results: Out of hospital treatment of hypoglycaemia is safe for most patients, but further studies are needed if positive improvements are to be made.

Discussion: There is potential for further research in this area and clarification is needed in the treat and release debate. A definitive set of protocols would be beneficial to the ambulance service.

$\mathrm{T}$ here is debate in the ambulance service as to whether patients suffering from hypoglycaemia need to be admitted to hospital after examination and treatment in the field. It is UK ambulance service protocol that patients who have experienced a hypoglycaemic event should be transported to hospital, but this is not compulsory. This review is concerned with the outcome of patients who are treated for hypoglycaemia out of hospital, and explores the current treat and release protocols used by the emergency services.

\section{METHODS}

Search methods

The information research process included:

- A literature search

- Research and location of periodical articles, books, and reviews

- Hand searching of various journals deemed relevant to the review

\section{Search strategies}

Various sources were searched to gather relevant keywords and MeSH (Medical Subject Headings) terms together. A

\begin{tabular}{|ll|}
\hline \multicolumn{2}{l}{$\begin{array}{l}\text { Table } 1 \\
\text { strategy used }\end{array}$} \\
\hline Search no & Search term used \\
\hline 1 & Patient: \\
2 & Refus: \\
3 & "treat and release" \\
4 & Treat: adj2 release: \\
5 & or/2-4 \\
6 & 1 and 5 \\
7 & Emergenc: \\
8 & (prehosp: or pre-hosp:) \\
9 & 7 and 8 \\
10 & 6 and 9 \\
\hline
\end{tabular}

search was set up on Medline. This search strategy was then adapted to each database (table 1).

Articles considered relevant to the review were retrieved and critically appraised according to a set of guidelines. The research findings were collated and discussed below.

\section{RESULTS}

Table 2 gives a summary of papers found in searches.

Research and evidence of treat and release protocols A six month retrospective study ${ }^{1}$ was carried out of patients who refused prehospital transport or treatment in an American EMS system. Seven per cent of refusals were associated with hypoglycaemia. In addition, leaving the scene against medical advice was associated with various factors including treated hypoglycaemia $(\mathrm{p}<0.05)$. Around the same time a report ${ }^{2}$ was published on "no-patient runs" (NPR). Carried out in Alaska, during the study period of 12 months, 2698 call outs (26.1\% of all call outs) consisted of (NPR, but of all NPR only $2.2 \%$ (59) were diabetes related. The study does not concentrate exclusively on diabetes related illness, but does reveal the extent of NPR in the ambulance service.

The literature search uncovered several other pieces of research discussing treat and release protocols and the outcome of hypoglycaemic patients. The earliest of these was a retrospective study ${ }^{3}$ carried out in the USA in 1991. The authors developed and tested criteria regarding which patients can safely be released without transportation to hospital. On the basis of their findings, they proposed five criteria that patients should meet before being released from prehospital care without the need for further treatment:

Table 2 Summary of papers found in searches

\begin{tabular}{ll}
\hline Total number of papers found & 241 \\
Papers ordered for review & 107 \\
Papers used in this article & 8 \\
\hline
\end{tabular}


- History of either type I or type II diabetes

- Pretreatment blood glucose of less than $4.4 \mathrm{mmol}$

- Post-treatment blood glucose greater than or equal to 4.4 mmol

- Return to normal mental status within 10 minutes of treatment

- Absence of complicating factors (renal dialysis, chest pain, alcohol, dyspnea)

In a further study, data were collected ${ }^{4}$ over an eight month period during which 132 hypoglycaemic patients refused transport after treatment with dextrose. Patients were followed up by a nurse three days after the hypoglycaemic event. Of these patients, $78 \%(n=103)$ were successfully contacted on the telephone, and $91 \%(n=94)$ had experienced no symptom recurrence. Of the nine patients who reported a recurrence of hypoglycaemia, six attended hospital, and three were admitted. One patient subsequently died from severe prolonged hypoglycaemia. It is interesting to note that no differences were found between hospital attendees and transport refusers in terms of clinical presentation or previous medical history. The authors conclude that transport to hospital should be "strongly encouraged" as there is a $10 \%$ chance of symptom recurrence.

Another study led to different conclusions. ${ }^{5}$ Over a seven month study period, 374 patients made a total of 571 calls regarding hypoglycaemia, which accounted for $5.2 \%$ of all ambulance runs. The aim of this study was to compare hypoglycaemic patients who attended hospital with those who refused transportation. Contrasting differences were found between the two groups. Patients transported to hospital were significantly older than those who refused $(\mathrm{p}<0.05)$. Among the patients taking oral hypoglycaemic agents, a significantly greater number of patients were transported than refused $(p<0.05)$. Ninety four per cent of the total number of patients were successfully treated and did not relapse. This rate is similar to the previous study. However, the authors are less cautious in their conclusion that out of hospital treatment for hypoglycaemia seems to be safe and effective independent of whether or not patients are transported to hospital.

Both studies discuss potential shortcomings and sources of bias. An incomplete response rate in the telephone survey was a possible source of bias. ${ }^{4}$ They were also concerned about the number of eligible patients not included in the study and suggest a larger scale multicentre study before any firm conclusions are drawn.

Two conference abstracts on treat and release protocols were identified (Wolford et $a l^{6}$ and A Billitier, SAEM 1998 Annual Meeting). A large study $(n=151)$ found that $66 \%$ of hypoglycaemic patients were transported to hospital and most of these were discharged from A\&E. ${ }^{6}$ They found that according to their criteria, $29 \%$ of patients were suitable for treatment and release. They suggest that further research is needed, a proposal echoed in another study (A Billitier, SAEM 1998 Annual Meeting). In this last study, patients $(n=34)$ were followed up 24 hours after a hypoglycaemic episode. Only three patients developed complications: two developed recurrent hypoglycaemia, which was successfully self treated. One patient was found unresponsive the following morning and required long term care for hypoglycaemic encephalopathy. Treat and release protocols were adjusted to avoid a recurrence of these situations. These changes incorporated the exclusion of patients who had experienced another hypoglycaemic episode in the past 30 days, and adding a request to the release form that the patient be woken every two hours. All study participants favoured making treat and release a permanent part of protocol. These short reports have raised some interesting issues, but as their authors suggest, the protocols may be in need of some "further refinement" ${ }^{16}$ and a randomised trial may be necessary to gain further insight into potential complication rates (A Billitier, SAEM 1998 Annual
Meeting). However, on the basis of a review ${ }^{7}$ of research and an extensive audit, the authors made some recommendations for protocols, which may serve as a starting point for further research. Some of these recommended protocols include the emphasis of clinical indications of hypoglycaemia to avoid misdiagnosis and under-treatment. Another suggests that insulin dependent patients can be left at home after a hypoglycaemic episode, providing a follow up letter is sent to the GP or primary care provider. These protocols, if applied, could help standardise the treatment of diabetic hypoglycaemia and ensure uniform practice throughout the ambulance service as a whole.

\section{DISCUSSION}

Hypoglycaemia is not considered a serious side effect of diabetes by many patients. They think it unnecessary to be admitted to hospital once the emergency services have treated the patient and the danger has passed. In many research papers follow up was either not carried out, carried out on a very small sample, or on patients who had to be re-admitted for treatment. The research does suggest that on the basis of the evidence, out of hospital treatment for hypoglycaemia is safe for about $90 \%$ of patients. A set of recommendations for the safe follow up of patients could include:

- Adding a request to the patient release form that the patient be woken every two hours for checks

- A follow up letter be sent to the GP or primary care provider after each hypoglycaemic episode

However, patients who fall into the following categories should be transported to hospital:

- Older (though a precise definition is not supplied)

- Taking oral hypoglycaemic medication

- Comorbid disorders

- No previous history of diabetes

- Post-treatment blood glucose is less than $4.4 \mathrm{mmol}$

- Normal mental status not achieved within 10 minutes of treatment

- Treatment with glucagon

- The presence of additional complicating factors including changed mental status, perceived head trauma, and symptoms indicating stroke or epilepsy

The success of the review is limited by the fact that much of the literature is outdated. Very little has been published recently that is directly relevant to emergency and prehospital treatment of hypoglycaemia. There is potential for further research in the form of a large multicentre randomised controlled trial. Considerable benefit could be gained from research into the treat and release protocols that exist already and whether or not they could be improved and standardised. Further research would be useful in establishing definitive protocols for use across the field of prehospital emergency medicine, and to affirm current rules and regulations regarding the treatment and release of people with diabetes who experience hypoglycaemic episodes in the community.

\section{ACKNOWLEDGEMENTS}

Malcolm Woollard for support and advice, given during the research project and in the writing of this article.

\section{Authors' affiliations \\ K Roberts, A Smith, Pre-hospital Emergency Research Unit (PERU), Lansdowne Hospital, Cardiff, UK}

Funding: The Wales Office for Research and Development for Health and Social Care.

Conflicts of interest: none. 


\section{REFERENCES}

1 Stark G, Hedges JR, Neely K. et al. Patients who initially refuse prehospital evaluation and/or therapy. Am J Emerg Med 1990:8:509-11.

2 Selden B, Schnitzer P, Nolan F. et al. The "no-patient" run: 2,698 patients evaluated but not transported by paramedics. Prehosp Dis Med $1991 ; 6: 135-42$.

3 Thompson RH, Wolford RW. Development and evaluation of criteria allowing paramedics to treat and release patients presenting with hypoglycaemia: a retrospective study. Prehosp Dis Med 1991;6:309-13.
4 Mechem CC, Kreshak AA, Barger J, et al. The short term outcome of hypoglycaemic patients who refuse ambulance transport after out-of-hospital therapy. Acad Emerg Med 1998;28:1630-4.

5 Socransky SJ, Pirrall RG, Rubin JM. Out-of-hospital treatment of hypoglycaemia: refusal of transport and patient outcome. Acad Emerg Med 1998;5:1080-5.

6 Wolford R, Tisol W, Vasilenko P, et al. Prospective evaluation of criteria allowing paramedics to treat and release patients presenting with hypoglycaemia. [Abstract.] Prehosp Dis Med 1996;109:S36.

7 South East Ambulance Clinical Audit Group. Treatment protocols for hypoglycaemic patients. London: SEACAG, 1999. 